\title{
INTEGRAL INEQUALITIES VIA GENERALIZED GEOMETRICALLY $r$-CONVEX FUNCTIONS
}

\author{
MUHAMMAD ASLAM NOOR ${ }^{1, *}$, KHALIDA INAYAT NOOR ${ }^{1}$ AND FARHAT SAFDAR ${ }^{2}$ \\ ${ }^{1}$ Department of Mathematics, COMSATS University Islamabad, Islamabad, Pakistan \\ ${ }^{2}$ Sardar Bahadur Khan Women's University, Quetta, Pakistan \\ *Corresponding author: noormaslam@gmail.com
}

\begin{abstract}
In this paper, we introduce and investigate a new class of generalized convex functions, called generalized geometrically $r$-convex functions. Some new Hermite-Hadamard integral inequalities via generalized geometrically $r$-convex functions have been established. Results proved in this paper can be viewed as new significant contributions in this area of research.
\end{abstract}

\section{INTRODUCTION}

Several branches of mathematical and engineering sciences has been developed by using the crucial and significant concepts of convex analysis and hence it becomes one of the most interesting and useful concept of mathematics for last few decades. There are mainly two aspects of the convex functions which have played very important and crucial part in the developments of various branches of pure and applied sciences. First aspect is concerned with differentiable convex functions. It is known that if a function $f$ is differentiable on the convex set $K$, then the $f$ is a convex function, if and only if, it satisfies the inequality

$$
\left\langle f^{\prime}(u), v-u\right\rangle \geq 0, \quad \forall v \in K
$$

Received 2018-06-10; accepted 2018-08-13; published 2018-11-02.

2010 Mathematics Subject Classification. 26D15, 26D10, 90C23.

Key words and phrases. generalized convex functions; generalized geometrically $r$-convex functions; Hermite-Hadamard type inequalities.

(C) 2018 Authors retain the copyrights of their papers, and all open access articles are distributed under the terms of the Creative Commons Attribution License. 
where $f^{\prime}($.$) is the Frechet differential of f$, which is called the variational inequality. Variational inequalities were introduced and studied by Stamapcchia [35] in potential theory. Variational inequalities can be viewed as natural generalization of the variational principles. It is remarkable that a wide class of unrelated problems, which arises in every branch of pure and applied sciences can be studied in the unified and general framework of variational inequalities and their variant forms. For the applications, formulation, dynamical systems, sensitivity analysis, numerical methods, error bounds and other aspects of the variational inequalities and optimization see $[3,4,8,16-21,35]$.

Hermite [13] and Hadamard [12] proved that a function $f$ is convex function on the interval $[a, b]$, if and only if, $f$ satisfies the inequality

$$
\left.\frac{a+b}{2}\right) \leq \frac{1}{b-a} \int_{a}^{b} f(x) d x \leq \frac{f(a)+f(b)}{2}, \quad \forall a, b \in[a, b]
$$

which is known as Hermite-Hadamard inequality and is one of the most important inequality. In recent years, much attention has been given to derive the Hermite-Hadamard type inequalities for various types of convex functions, see $[6,11,14,15,22-33]$.

The concept of convexity has been extended and generalized in several directions using new and innovative techniques, see $[1,2,3,5,7,11,12,13]$ and the references therein. Pearce et. al [11] introduced the class of r-convex functions. Several authors have derived Hermite-Hadamard type inequalities for various classes of $r$-convex functions, see [22, 28,31-33]. Gordji et al. [9] introduced an important class of convex functions involving the bifunction, which is called generalized( $\varphi$-convex $)$ convex function. These generalized convex functions are nonconvex functions. For recent developments, see $[5,6,7,14,15,16,17,18,20,21,22]$ and the references therein.

Inspired and motivated by the ongoing research in this field, we introduce a new class of generalized convex function, which is known as generalized geometrically $r$-convex function. We derive some new HermiteHadamard integral inequalities for these nonconvex functions. Some special cases are discussed, which can be obtained from our new results. Using the technique and ideas of this paper, one may obtain HermiteHadamard type integral inequalities for other classes of convex functions and their variant forms.

\section{Preliminaries}

Let $I=[a, b]$ be an interval in real line $\mathbb{R}$. Let $f: I \rightarrow \mathbb{R}$ be a continuous function and $\eta(\cdot, \cdot): \mathbb{R} \times \mathbb{R} \rightarrow \mathbb{R}$ be a continuous bifunction. First of all, we have the following well known and new concepts. 
Definition 2.1. [9]. A function $f: I=[a, b] \rightarrow \mathbb{R}$ is said to be generalized convex function with respect to a bifunction $\eta(\cdot, \cdot): \mathbb{R} \times \mathbb{R} \rightarrow \mathbb{R}$, if

$$
f((1-t) a+t b) \leq(1-t) f(a)+t[f(a)+\eta(f(b), f(a))], \forall a, b \in I, t \in[0,1] .
$$

Definition 2.2. [32]. A function $f: I=[a, b] \rightarrow \mathbb{R}$ is said to be generalized $r$-convex function with respect to a bifunction $\eta(\cdot, \cdot): \mathbb{R} \times \mathbb{R} \rightarrow \mathbb{R}$, if $\forall a, b \in I, t \in[0,1]$

$$
f((1-t) a+t b) \leq \begin{cases}\left\{(1-t)[f(a)]^{r}+t[f(a)+\eta(f(b), f(a))]^{r}\right\}^{\frac{1}{r}}, & r \neq 0, \\ {[f(a)]^{1-t}[f(a)+\eta(f(b), f(a))]^{t},} & r=0 .\end{cases}
$$

If $\eta(f(b)-f(a))=f(b)-f(a)$, then, the Definition 2.2 reduces to

Definition 2.3. [11]. A function $f: I=[a, b] \rightarrow \mathbb{R}$ is said to be $r$-convex function, if

$$
f((1-t) a+t b) \leq \begin{cases}\left\{(1-t)[f(a)]^{r}+t[f(b)]^{r}\right\}^{\frac{1}{r}}, & r \neq 0, \\ {[f(a)]^{1-t}[f(b)]^{t},} & r=0 .\end{cases}
$$

Note that for $r=1$, we have classical convex functions and for $r=0$, we have log-convex functions.

Definition 2.4. [33]. The set $I \subset \mathbb{R}_{+}$is said to be geometrically convex set, if

$$
a^{1-t} b^{t} \in I, \quad \forall a, b \in I, t \in[0,1] .
$$

Definition 2.5. [33]. A function $f: I \subset \mathbb{R}_{+}=(0, \infty) \rightarrow \mathbb{R}$ is said to be geometrically convex, if

$$
f\left(a^{1-t} b^{t}\right) \leq(1-t) f(a)+t(f(b)), \quad \forall a, b \in I, t \in[0,1]
$$

We now define a new concept of generalized geometrically $r$-convex functions.

Definition 2.6. . A function $f: I=[a, b] \rightarrow \mathbb{R}$ is said to be generalized geometrically $r$-convex function with respect to a bifunction $\eta(\cdot, \cdot): \mathbb{R} \times \mathbb{R} \rightarrow \mathbb{R}$, if $\forall a, b \in I, t \in[0,1]$

$$
f\left(a^{1-t} b^{t}\right) \leq \begin{cases}\left\{(1-t)[f(a)]^{r}+t[f(a)+\eta(f(b), f(a))]^{r}\right\}^{\frac{1}{r}}, & r \neq 0, \\ {[f(a)]^{1-t}[f(a)+\eta(f(b), f(a))]^{t},} & r=0 .\end{cases}
$$

If $t=\frac{1}{2}$, then definition 2.6 reduces to

$$
f(\sqrt{a b}) \leq \begin{cases}\left\{\frac{[f(a)]^{r}+[f(a)+\eta(f(b), f(a))]^{r}}{2}\right\}^{\frac{1}{r}}, & r \neq 0, \\ \sqrt{[f(a)][f(a)+\eta(f(b), f(a))]}, & r=0 .\end{cases}
$$


The function $f$ is called generalized geometrically Jensen $r$-convex function.

If $\eta(f(b), f(a))=f(b)-f(a)$, then Definition 2.6 reduces to a new concept.

Definition 2.7. . A function $f: I=[a, b] \rightarrow \mathbb{R}$ is said to be generalized geometrically $r$-convex function with respect to a bifunction $\eta(\cdot, \cdot): \mathbb{R} \times \mathbb{R} \rightarrow \mathbb{R}$, if $\forall a, b \in I, t \in[0,1]$

$$
f\left(a^{1-t} b^{t}\right) \leq \begin{cases}\left\{(1-t)[f(a)]^{r}+t[f(b)]^{r}\right\}^{\frac{1}{r}}, & r \neq 0, \\ {[f(a)]^{1-t}[f(b)]^{t},} & r=0 .\end{cases}
$$

If $t=\frac{1}{2}$, then

$$
f(\sqrt{a b}) \leq \begin{cases}\left\{\frac{[f(a)]^{r}+[f(b)]^{r}}{2}\right\}^{\frac{1}{r}}, & r \neq 0, \\ \sqrt{[f(a) f(b)]}, & r=0 .\end{cases}
$$

The function $f$ is called generalized geometrically Jensen $r$-convex function.

The generalized logarithmic means of order $r$ of positive numbers $a, b$ is defined by

$$
L_{r}(a, b)= \begin{cases}\frac{r}{r+1} \frac{a^{r+1}-b^{r+1}}{a^{r}-b^{r}}, & r \neq 0,-1, a \neq b, \\ \frac{a-b}{\log a-\log b}, & r=0, a \neq b, \\ a b \frac{\log a-\log b}{a-b}, & r=-1, a \neq b, \\ a, & a=b .\end{cases}
$$

Definition 2.8. The beta function, also called the Euler integral of the first kind, is defined as

$$
\beta(x, y)=\int_{0}^{1} t^{x-1}(1-t)^{y-1} \mathrm{~d} t=\frac{\Gamma(x) \Gamma(y)}{\Gamma(x+y)}, \quad x, y>0
$$

where $\Gamma($.$) is a Gamma function.$

\section{MAin Results}

In this section, we establish several new integral inequalities of Hermite-Hadamard type for generalized geometrically $r$-convex functions.

Theorem 3.1. Let $f: I \rightarrow \mathbb{R}$ be generalized geometrically $r$-convex function on $I$. Then for $0<r \leq 1$, we have

$$
\frac{1}{\log b-\log a} \int_{a}^{b} \frac{1}{x} f(x) \mathrm{d} x \leq\left(\frac{r}{r+1}\right)\left\{\left([f(a)]^{r}+[f(a)+\eta(f(b), f(a))]^{r}\right)\right\}^{\frac{1}{r}}
$$


Proof. Let $f$ be generalized geometrically $r$-convex function on $I$. Then, $\forall a, b \in I, t \in[0,1]$, we have

$$
f\left(a^{1-t} b^{t}\right) \leq\left\{(1-t)[f(a)]^{r}+t[f(a)+\eta(f(b), f(a))]^{r}\right\}^{\frac{1}{r}} .
$$

Using Minkowski's inequality and the fact that $f$ is generalized geometrically $r$-convex function, we have

$$
\begin{aligned}
\frac{1}{\log b-\log a} \int_{a}^{b} \frac{1}{x} f(x) \mathrm{d} x= & \int_{0}^{1} f\left(a^{1-t} b^{t}\right) \mathrm{d} t \\
\leq & \int_{0}^{1}\left\{(1-t)[f(a)]^{r}+t[f(a)+\eta(f(b), f(a))]^{r}\right\}^{\frac{1}{r}} \mathrm{~d} t \\
\leq & \left\{\left(\int_{0}^{1}(1-t)^{\frac{1}{r}}[f(a)] \mathrm{d} t\right)^{r}\right. \\
& \left.+\left(\int_{0}^{1} t^{\frac{1}{r}}[f(a)+\eta(f(b), f(a))] \mathrm{d} t\right)^{r}\right\}^{\frac{1}{r}} \\
= & \left\{\left(\frac{r}{r+1}\right)^{r}\left([f(a)]^{r}+[f(a)+\eta(f(b), f(a))]^{r}\right)\right\}^{\frac{1}{r}} \\
= & \left(\frac{r}{r+1}\right)\left\{\left([f(a)]^{r}+[f(a)+\eta(f(b), f(a))]^{r}\right)\right\}^{\frac{1}{r}},
\end{aligned}
$$

which is the required result.

Corollary 3.1. If $\eta(f(b), f(a))=f(b)-f(a)$, then, under the assumptions of Theorem 3.1, we have a new result.

$$
\frac{1}{\log b-\log a} \int_{a}^{b} \frac{1}{x} f(x) \mathrm{d} x \leq\left(\frac{r}{r+1}\right)\left\{\left([f(a)]^{r}+[f(b)]^{r}\right)\right\}^{\frac{1}{r}} .
$$

Theorem 3.2. Let $f: I \rightarrow \mathbb{R}$ be generalized geometrically $r$-convex function on $I$. Then for $0<r \leq 1$, we have

$$
\begin{aligned}
& 2[f(\sqrt{a b})]^{r}-\frac{1}{(\log b-\log a)} \int_{a}^{b}\left[\frac{1}{x} f(x)+\eta\left(f\left(\frac{a b}{x}\right), \frac{1}{x} f(x)\right)\right]^{r} \mathrm{~d} x \\
\leq & \frac{1}{\log b-\log a} \int_{a}^{b}[f(x)]^{r} \mathrm{~d} x \\
\leq & \frac{\left\{[f(a)]^{r}+[f(b)]^{r}\right\}}{4}+\frac{1}{4}\left([f(a)+\eta(f(b), f(a))]^{r}+[f(b)+\eta(f(a), f(b))]^{r}\right),
\end{aligned}
$$

Proof. Let $f$ be generalized geometrically $r$-convex function on $I$. Then, $\forall a, b \in I, t \in[0,1]$, we have

$$
f\left(a^{1-t} b^{t}\right) \leq\left\{(1-t)[f(a)]^{r}+t[f(a)+\eta(f(b), f(a))]^{r}\right\}^{\frac{1}{r}} .
$$

Using (2.1) and substituting $x=a^{1-t} b^{t}$ and $y=a^{t} b^{1-t}$, we have

$$
[f(\sqrt{a b})]^{r} \leq\left\{\frac{\left[f\left(a^{1-t} b^{t}\right)\right]^{r}+\left[f\left(a^{1-t} b^{t}\right)+\eta\left(f\left(a^{t} b^{1-t}\right), f\left(a^{1-t} b^{t}\right)\right)\right]^{r}}{2}\right\} .
$$


Integrating the above inequality with respect to $t$ on $[0,1]$, we have

$$
\begin{aligned}
{[f(\sqrt{a b})]^{r} \leq } & \frac{1}{2} \int_{0}^{1}\left\{\left[f\left(a^{1-t} b^{t}\right)\right]^{r}+\left[f\left(a^{1-t} b^{t}\right)\right.\right. \\
& \left.\left.+\eta\left(f\left(a^{t} b^{1-t}\right), f\left(a^{1-t} b^{t}\right)\right)\right]^{r}\right\} \mathrm{d} t \\
= & \frac{1}{2(\log b-\log a)} \int_{a}^{b} \frac{1}{x}\left\{[f(x)]^{r}+\left[f(x)+\eta\left(f\left(\frac{a b}{x}\right), f(x)\right)\right]^{r}\right\} \mathrm{d} x .
\end{aligned}
$$

This implies

$$
\begin{aligned}
& 2[f(\sqrt{a b})]^{r}-\frac{1}{(\log b-\log a)} \int_{a}^{b} \frac{1}{x}\left[f(x)+\eta\left(f\left(\frac{a b}{x}\right), f(x)\right)\right]^{r} \mathrm{~d} x \\
\leq & \frac{1}{(\log b-\log a)} \int_{a}^{b} \frac{1}{x}[f(x)]^{r} \mathrm{~d} x .
\end{aligned}
$$

Consider

$$
\begin{aligned}
& {\left[f\left(a^{1-t} b^{t}\right)\right]^{r} \leq\left\{(1-t)[f(a)]^{r}+t[f(a)+\eta(f(b), f(a))]^{r}\right\} .} \\
& {\left[f\left(a^{t} b^{1-t}\right)\right]^{r} \leq\left\{(1-t)[f(b)]^{r}+t[f(b)+\eta(f(a), f(b))]^{r}\right\} .}
\end{aligned}
$$

Adding (3.2) and (3.3), we have

$$
\begin{aligned}
{\left[f\left(a^{1-t} b^{t}\right)^{r}+\left[f\left(a^{t} b^{1-t}\right)\right]^{r} \leq\right.} & \left\{(1-t)[f(a)]^{r}+t[f(a)+\eta(f(b), f(a))]^{r}\right\} \\
& +\left\{(1-t)[f(b)]^{r}+t[f(b)+\eta(f(a), f(b))]^{r}\right\} .
\end{aligned}
$$

Integrating the above inequality with respect to $t$ on $[0,1]$, we have

$$
\begin{aligned}
\frac{2}{\log b-\log a} \int_{a}^{b} f^{r}(x) \mathrm{d} x \leq & \int_{0}^{1}\left\{(1-t)\left([f(a)]^{r}+[f(b)]^{r}\right)+t\left([f(a)+\eta(f(b), f(a))]^{r}\right.\right. \\
& \left.\left.+[f(b)+\eta(f(a), f(b))]^{r}\right)\right\} \mathrm{d} t
\end{aligned}
$$

which implies that

$$
\begin{aligned}
\frac{1}{\log b-\log a} \int_{a}^{b}[f(x)]^{r} \mathrm{~d} x \leq & \left\{\frac{\left\{[f(a)]^{r}+[f(b)]^{r}\right\}}{4}+\frac{1}{4}\left([f(a)+\eta(f(b), f(a))]^{r}\right.\right. \\
& \left.\left.+[f(b)+\eta(f(a), f(b))]^{r}\right)\right\} .
\end{aligned}
$$

Combining (3.1) and (3.4), we have

$$
\begin{aligned}
& 2[f(\sqrt{a b})]^{r}-\frac{1}{(\log b-\log a)} \int_{a}^{b}\left[\frac{1}{x} f(x)+\eta\left(f\left(\frac{a b}{x}\right), \frac{1}{x} f(x)\right)\right]^{r} \mathrm{~d} x \\
\leq & \frac{1}{\log b-\log a} \int_{a}^{b}[f(x)]^{r} \mathrm{~d} x \\
\leq & \frac{\left\{[f(a)]^{r}+[f(b)]^{r}\right\}}{4}+\frac{1}{4}\left([f(a)+\eta(f(b), f(a))]^{r}+[f(b)+\eta(f(a), f(b))]^{r}\right),
\end{aligned}
$$


which is the required result.

Corollary 3.2. If $\eta(f(b), f(a))=f(b)-f(a)$, then, under the assumptions of Theorem 3.2, we have a new result.

$$
[f(\sqrt{a b})]^{r} \leq \frac{1}{\log b-\log a} \int_{a}^{b}[f(x)]^{r} \mathrm{~d} x \leq\left\{\frac{[f(a)]^{r}+[f(b)]^{r}}{2}\right\}
$$

Theorem 3.3. Let $f: I \rightarrow \mathbb{R}$ be generalized geometrically $r$-convex function on $I$ and $r \geq 0$, then

$$
\frac{1}{\log b-\log a} \int_{a}^{b} \frac{1}{x} f(x) \mathrm{d} x \leq \begin{cases}\left(\frac{r}{r+1}\right)\left\{\frac{\left([f(a)+\eta(f(b), f(a))]^{r+1}-[f(a)]^{r+1}\right)}{[f(a)+\eta(f(b), f(a))]^{r}-[f(a)]^{r}}\right\}, & r \neq 0 \\ \frac{[f(a)+\eta(f(b), f(a))]-[f(a)]}{\log [f(a)+\eta(f(b), f(a))]-\log [f(a)]}, & r=0 .\end{cases}
$$

Proof. First, let $r>0$ and $f$ be generalized geometrically $r$-convex function on $I$. Then $\forall a, b \in I, t \in[0,1]$, we have

$$
\begin{aligned}
\frac{1}{\log b-\log a} \int_{a}^{b} \frac{1}{x} f(x) \mathrm{d} x & =\int_{0}^{1} f\left(a^{1-t} b^{t}\right) \mathrm{d} t \\
& \leq \int_{0}^{1}\left\{(1-t)[f(a)]^{r}+t[f(a)+\eta(f(b), f(a))]^{r}\right\}^{\frac{1}{r}} \mathrm{~d} t
\end{aligned}
$$

Substituting $u=\left[(1-t)[f(a)]^{r}+t[f(a)+\eta(f(b), f(a))]^{r}\right]$ in (3.5), we have

$$
\begin{aligned}
\frac{1}{\log b-\log a} \int_{a}^{b} \frac{1}{x} f(x) \mathrm{d} x & \leq \frac{1}{[f(a)+\eta(f(b), f(a))]^{r}-[f(a)]^{r}} \int_{[f(a)]^{r}}^{[f(a)+\eta(f(b), f(a))]^{r}} u^{\frac{1}{r}} \mathrm{~d} u \\
& =\left(\frac{r}{r+1}\right)\left\{\frac{\left([f(a)+\eta(f(b), f(a))]^{r+1}-[f(a)]^{r+1}\right)}{[f(a)+\eta(f(b), f(a))]^{r}-[f(a)]^{r}}\right\}
\end{aligned}
$$

For $r=0$, we have

$$
f\left(a^{1-t} b^{t}\right) \leq\left\{[f(a)]^{1-t}[f(a) \eta(f(b), f(a))]^{t}\right\} .
$$

Hence we have,

$$
\begin{aligned}
\frac{1}{\log b-\log a} \int_{a}^{b} \frac{1}{x} f(x) \mathrm{d} x & =\int_{0}^{1} f\left(a^{1-t} b^{t}\right) \mathrm{d} t \\
& \leq \int_{0}^{1}\left\{[f(a)]^{1-t}[f(a)+\eta(f(b), f(a))]^{t}\right\} \mathrm{d} t \\
& =[f(a)] \int_{0}^{1}\left\{\frac{[f(a)+\eta(f(b), f(a))]}{[f(a)]}\right\}^{t} \mathrm{~d} t \\
& =\frac{[f(a)+\eta(f(b), f(a))]-[f(a)]}{\log [f(a)+\eta(f(b), f(a))]-\log [f(a)]}
\end{aligned}
$$

which is the required result. 
Corollary 3.3. If $\eta(f(b), f(a))=f(b)-f(a)$, then, under the assumptions of Theorem 3.3, we have a new result.

$$
\frac{1}{\log b-\log a} \int_{a}^{b} \frac{1}{x} f(x) \mathrm{d} x \leq \begin{cases}\left(\frac{r}{r+1}\right)\left\{\frac{[f(b)]^{r+1}-[f(a)]^{r+1}}{[f(b)]^{r}-[f(a)]^{r}}\right\}=L_{r}(f(a), f(b)), & r \neq 0 \\ \left\{\frac{[f(b)-f(a)]}{\log [f(b)]-\log [f(a)]}\right\}=L(f(a), f(b)), & r=0 .\end{cases}
$$

Theorem 3.4. Let $f: I \rightarrow \mathbb{R}$ be generalized geometrically $r$-convex function on $I$ and $r \geq 0$, then

$$
\frac{1}{\log b-\log a} \int_{a}^{b} \frac{1}{x} f(x) \mathrm{d} x \leq\left\{\begin{array}{l}
f(a), r \neq 0, f(a)=f(b) \\
\frac{\left[\log [f(a)+\eta(f(b), f(a))]^{-1}-\log [f(a)]^{-1}\right]}{[f(a)+\eta(f(b), f(a))]^{-1}-[f(a)]^{-1}}, r=-1 .
\end{array}\right.
$$

Proof. First, let $r>0, f$ be generalized geometrically $r$-convex function on $I$ and $f(a)=f(b)$. Then $\forall a, b \in I, t \in[0,1]$, we have

$$
\begin{aligned}
\frac{1}{\log b-\log a} \int_{a}^{b} \frac{1}{x} f(x) \mathrm{d} x & =\int_{0}^{1} f\left(a^{1-t} b^{t}\right) \mathrm{d} t \\
& \leq \int_{0}^{1}\left\{(1-t)[f(a)]^{r}+t[f(a)+\eta(f(a), f(a))]^{r}\right\}^{\frac{1}{r}} \mathrm{~d} t \\
& =f(a)
\end{aligned}
$$

For $r=-1$ and $f(a) \neq f(b)$, we have

$$
\begin{aligned}
\frac{1}{\log b-\log a} \int_{a}^{b} \frac{1}{x} f(x) \mathrm{d} x & =\int_{0}^{1} f\left(a^{1-t} b^{t}\right) \mathrm{d} t \\
& \leq \int_{0}^{1}\left\{(1-t)[f(a)]^{-1}+t[f(a)+\eta(f(b), f(a))]^{-1}\right\}^{-1} \mathrm{~d} t \\
& =\frac{1}{[f(a)+\eta(f(b), f(a))]^{-1}-[f(a)]^{-1}} \int_{[f(a)]^{-1}}^{[f(a)+\eta(f(b), f(a))]^{-1}} \frac{1}{u} \mathrm{~d} u \\
& =\frac{\left[\log [f(a)+\eta(f(b), f(a))]^{-1}-\log [f(a)]^{-1}\right]}{[f(a)+\eta(f(b), f(a))]^{-1}-[f(a)]^{-1}}
\end{aligned}
$$

which is the required result.

Corollary 3.4. If $\eta(f(b), f(a))=f(b)-f(a)$, then, under the assumptions of Theorem 3.4, we have a new result.

$$
\frac{1}{\log b-\log a} \int_{a}^{b} \frac{1}{x} f(x) \mathrm{d} x \leq\left\{\begin{array}{l}
f(a), r \neq 0, f(a)=f(b), \\
f(a) f(b) \frac{\log [f(b)]-\log [f(a)]}{f(b)-f(a)}=L_{-1}(f(a), f(b), \quad r=-1 .
\end{array}\right.
$$


Theorem 3.5. Let $f, g: I \rightarrow \mathbb{R}$ be generalized $r_{1}$-convex function and generalized $r_{2}$-convex functions respectively on $I$. Then for $r_{1}>0, r_{2}>0>$, we have

$$
\begin{aligned}
\frac{1}{\log b-\log a} \int_{a}^{b} \frac{1}{x} f(x) g(x) \mathrm{d} x \leq & \left(\frac{r}{r+1}\right)\left\{\left([f(a)]^{r_{1}}+[f(a)+\eta(f(b), f(a))]^{r_{1}}\right)\right\}^{\frac{2}{r_{1}}} \\
& +\left(\frac{r}{r+1}\right)\left\{\left([g(a)]^{r_{2}}[g(a)+\eta(g(b), g(a))]^{r_{2}}\right)\right\}^{\frac{2}{r_{2}}} .
\end{aligned}
$$

Proof. Let $f, g: I \rightarrow \mathbb{R}$ be generalized $r_{1}$-convex function and generalized $r_{2}$-convex functions respectively on $I$ with $\left(r_{1}>0, r_{2}>0\right)$. Then $\forall a, b \in I, t \in[0,1]$, we have

$$
\begin{aligned}
& f\left(a^{1-t} b^{t}\right) \leq\left\{(1-t)[f(a)]^{r_{1}}+t[f(a)+\eta(f(b), f(a))]^{r_{1}}\right\}^{\frac{1}{r_{1}}} \\
& g\left(a^{1-t} b^{t}\right) \leq\left\{(1-t)[g(a)]^{r_{2}}+t[g(a)+\eta(g(b), g(a))]^{r_{2}}\right\}^{\frac{1}{r_{2}}}
\end{aligned}
$$

Using Cauchy's and Minkowski's inequalities and the fact that $f$ and $g$ are generalized $r_{1}$ and $r_{2}$-convex functions, we have

$$
\begin{aligned}
& \frac{1}{\log b-\log a} \int_{a}^{b} \frac{1}{x} f(x) g(x) \mathrm{d} x \\
= & \int_{0}^{1} f\left(a^{1-t} b^{t}\right) g\left(a^{1-t} b^{t}\right) \mathrm{d} t \\
\leq & \int_{0}^{1}\left\{(1-t)[f(a)]^{r_{1}}+t[f(a)+\eta(f(b), f(a))]^{r_{1}}\right\}^{\frac{1}{r_{1}}} \\
& \left\{(1-t)[g(a)]^{r_{2}}+t[g(a)+\eta(g(b), g(a))]^{r_{2}}\right\}^{\frac{1}{r_{2}}} \mathrm{~d} t \\
\leq & \frac{1}{2} \int_{0}^{1}\left\{(1-t)[f(a)]^{r_{1}}+t[f(a)+\eta(f(b), f(a))]^{r_{1}}\right\}^{\frac{2}{r_{1}}} \mathrm{~d} t \\
& +\frac{1}{2} \int_{0}^{1}\left\{(1-t)[g(a)]^{r_{2}}+t[g(a)+\eta(g(b), g(a))]^{r_{2}}\right\}^{\frac{2}{r_{2}}} \mathrm{~d} t \\
\leq & \frac{1}{2}\left\{\left(\int_{0}^{1}(1-t)^{\frac{2}{r_{1}}}[f(a)]^{2} \mathrm{~d} t\right)\right)^{\frac{r_{1}}{2}} \\
& \left.+\left(\int_{0}^{1} t^{\frac{2}{r_{1}}}[f(a)+\eta(f(b), f(a))]^{2} \mathrm{~d} t\right)^{\frac{r_{1}}{2}}\right\}^{\frac{2}{r_{1}}} \\
+ & \frac{1}{2}\left\{\left(\int_{0}^{1}(1-t)^{\frac{2}{r_{2}}}[g(a)]^{2} \mathrm{~d} t\right)\right)^{\frac{r_{2}}{2}} \\
+ & \left.\left(\int_{0}^{1} t^{\frac{2}{r_{2}}}[g(a)+\eta(g(b), g(a))]^{2} \mathrm{~d} t\right)^{\frac{r_{2}}{2}}\right\}^{\frac{2}{r_{2}}}
\end{aligned}
$$




$$
\begin{aligned}
= & \left\{\left(\frac{r}{r+1}\right)^{\frac{r_{1}}{2}}\left([f(a)]^{r_{1}}+[f(a)+\eta(f(b), f(a))]^{r_{1}}\right)\right\}^{\frac{2}{r_{1}}} \\
& +\left\{\left(\frac{r}{r+1}\right)^{\frac{r_{2}}{2}}\left([g(a)]^{r_{2}}[g(a)+\eta(g(b), g(a))]^{r_{2}}\right)\right\}^{\frac{2}{r_{2}}} \\
= & \left(\frac{r}{r+1}\right)\left\{\left([f(a)]^{r_{1}}+[f(a)+\eta(f(b), f(a))]^{r_{1}}\right)\right\}^{\frac{2}{r_{1}}} \\
& +\left(\frac{r}{r+1}\right)\left\{\left([g(a)]^{r_{2}}[g(a)+\eta(g(b), g(a))]^{r_{2}}\right)\right\}^{\frac{2}{r_{2}}},
\end{aligned}
$$

which is the required result.

Corollary 3.5. If $\eta(f(b), f(a))=f(b)-f(a)$, then, under the assumptions of Theorem 3.5, we have

$$
\begin{aligned}
\frac{1}{\log b-\log a} \int_{a}^{b} \frac{1}{x} f(x) g(x) \mathrm{d} x \leq & \left(\frac{r}{r+1}\right)\left\{\left([f(a)]^{r_{1}}+[f(b)]^{r_{1}}\right)\right\}^{\frac{2}{r_{1}}} \\
& +\left(\frac{r}{r+1}\right)\left\{\left([g(a)]^{r_{2}}+[g(b)]^{r_{2}}\right)\right\}^{\frac{2}{r_{2}}}
\end{aligned}
$$

Theorem 3.6. Let $f, g: I \rightarrow \mathbb{R}$ be generalized $r_{1}$-convex function and generalized $r_{2}$-convex functions respectively on $I$. Then for $r_{1}>0, r_{2}>0$ and $\frac{1}{r_{1}}+\frac{1}{r_{2}}=1$, we have

$$
\begin{aligned}
\frac{1}{\log b-\log a} \int_{a}^{b} \frac{1}{x} f(x) g(x) \mathrm{d} x \leq & \frac{1}{2}\left\{\left([f(a)]^{r_{1}}+[f(a)+\eta(f(b), f(a))]^{r_{1}}\right)^{\frac{1}{r_{1}}}\right. \\
& \left.\left([g(a)]^{r_{2}}+[g(a)+\eta(g(b), g(a))]^{r_{2}}\right)^{\frac{1}{r_{2}}}\right\}
\end{aligned}
$$

Proof. Let $f, g: I \rightarrow \mathbb{R}$ be generalized $r_{1}$-convex function and generalized $r_{2}$-convex functions respectively on $I$ with $\left(r_{1}>0, r_{2}>0\right)$. Then $\forall a, b \in I, t \in[0,1]$, we have

$$
\begin{aligned}
& f\left(a^{1-t} b^{t}\right) \leq\left\{(1-t)[f(a)]^{r_{1}}+t[f(a)+\eta(f(b), f(a))]^{r_{1}}\right\}^{\frac{1}{r_{1}}} \\
& g\left(a^{1-t} b^{t}\right) \leq\left\{(1-t)[g(a)]^{r_{2}}+t[g(a)+\eta(g(b), g(a))]^{r_{2}}\right\}^{\frac{1}{r_{2}}} .
\end{aligned}
$$


Using Holder's inequality and the fact that $f$ and $g$ are generalized $r_{1}$ and $r_{2}$-convex functions, we have

$$
\begin{aligned}
& \frac{1}{\log b-\log a} \int_{a}^{b} \frac{1}{x} f(x) g(x) \mathrm{d} x \\
= & \int_{0}^{1} f\left(a^{1-t} b^{t}\right) g\left(a^{1-t} b^{t}\right) \mathrm{d} t \\
\leq & \int_{0}^{1}\left\{(1-t)[f(a)]^{r_{1}}+t[f(a)+\eta(f(b), f(a))]^{r_{1}}\right\}^{\frac{1}{r_{1}}} \\
& \left\{(1-t)[g(a)]^{r_{2}}+t[g(a)+\eta(g(b), g(a))]^{r_{2}}\right\}^{\frac{1}{r_{2}}} \mathrm{~d} t \\
\leq & \left\{\int_{0}^{1}(1-t)[f(a)]^{r_{1}}+t[f(a)+\eta(f(b), f(a))]^{r_{1}} \mathrm{~d} t\right\}^{\frac{1}{r_{1}}} \\
= & \left\{\int_{0}^{1}(1-t)[g(a)]^{r_{2}}+t[g(a)+\eta(g(b), g(a))]^{r_{2}} \mathrm{~d} t\right\}^{\frac{1}{r_{2}}} \\
& \left.\left\{(f(a)]^{r_{1}} \int_{0}^{1}(1-t) \mathrm{d} t+[f(a)+\eta(f(b), f(a))]^{r_{1}} \int_{0}^{1} t \mathrm{~d} t\right)\right\}^{\frac{1}{r_{1}}} \\
& \left\{\left([g(a)]^{r_{2}} \int_{0}^{1}(1-t) \mathrm{d} t+[g(a)+\eta(g(b), g(a))]^{r_{2}} \int_{0}^{1} t \mathrm{~d} t\right)\right\}^{\frac{1}{r_{2}}} \\
& \frac{1}{2}\left\{\left([f(a)]^{r_{1}}+[f(a)+\eta(f(b), f(a))]^{r_{1}}\right)\right. \\
& \left.\left([g(a)]^{\frac{1}{r_{1}}}+\left[g^{r_{2}}(a)+\eta(g(b), g(a))\right]^{r_{2}}\right)^{\frac{1}{r_{2}}}\right\},
\end{aligned}
$$

which is the required result.

Corollary 3.6. If $\eta(f(b), f(a))=f(b)-f(a)$, then, under the assumptions of Theorem 3.6, we have a new result.

$$
\frac{1}{\log b-\log a} \int_{a}^{b} \frac{1}{x} f(x) g(x) \mathrm{d} x \leq \frac{\left\{\left([f(a)]^{r_{1}}+[f(b)]^{r_{1}}\right)^{\frac{1}{r_{1}}}\left([g(a)]^{r_{2}}+[g(b)]^{r_{2}}\right)^{\frac{1}{r_{1}}}\right\}}{2} .
$$

Theorem 3.7. Let $f, g: I \rightarrow \mathbb{R}$ be generalized geometrically $r$-convex function on $I$. Then for $r>0$, we have

$$
\left(\frac{1}{\log b-\log a} \int_{a}^{b} \frac{1}{x} f(x) g(x) \mathrm{d} x\right)^{r} \leq\left\{M(a, b)\left(\frac{r}{r+2}\right)^{r}+N(a, b)\left(\beta\left(\frac{1}{r}+1, \frac{1}{r}+1\right)\right)^{r}\right\} .
$$

where

$$
\begin{aligned}
& M(a, b)=\left([f(a)]^{r}[g(a)]^{r}+[f(a)+\eta(f(b), f(a))]^{r}[g(a)+\eta(g(b), g(a))]^{r}\right) \\
& N(a, b)=\left([f(a)]^{r}[g(a)+\eta(g(b), g(a))]^{r}+[g(a)]^{r}[f(a)+\eta(f(b), f(a))]^{r}\right),
\end{aligned}
$$


and $\beta(\cdot, \cdot)$ is the Beta function.

Proof. Let $f, g$ be two generalized geometrically $r$-convex functions on $I$. Then $\forall a, b \in I, t \in[0,1]$, we have

$$
\begin{aligned}
& f\left(a^{1-t} b^{t}\right) \leq\left\{(1-t)[f(a)]^{r}+t[f(a)+\eta(f(b), f(a))]^{r}\right\}^{\frac{1}{r}} \\
& g\left(a^{1-t} b^{t}\right) \leq\left\{(1-t)[g(a)]^{r}+t[g(a)+\eta(g(b), g(a))]^{r}\right\}^{\frac{1}{r}} .
\end{aligned}
$$

Using Minkowski's inequality and the fact that $f$ and $g$ are generalized geometrically $r$-convex functions, we have

$$
\begin{aligned}
& \left(\frac{1}{\log b-\log a} \int_{a}^{b} \frac{1}{x} f(x) g(x) \mathrm{d} x\right)^{r} \\
& =\left(\int_{0}^{1} f\left(a^{1-t} b^{t}\right) g\left(a^{1-t} b^{t}\right) \mathrm{d} t\right)^{r} \\
& \leq\left\{\int _ { 0 } ^ { 1 } \left((1-t)[f(a)]^{r}+t[f(a)+\eta(f(b), f(a))]^{r}\right.\right. \\
& \left.\left.(1-t)[g(a)]^{r}+t[g(a)+\eta(g(b), g(a))]^{r}\right)^{\frac{1}{r}} \mathrm{~d} t\right\}^{r} \\
& =\left\{\int _ { 0 } ^ { 1 } \left((1-t)^{2}[f(a)]^{r}[g(a)]^{r}\right.\right. \\
& +t(1-t)\left([f(a)]^{r}[g(a)+\eta(g(b), g(a))]^{r}+[g(a)]^{r}[f(a)+\eta(f(b), f(a))]^{r}\right) \\
& \left.+t^{2}\left([f(a)+\eta(f(b), f(a))]^{r}[g(a)+\eta(g(b), g(a))]^{r}\right)\right\}^{r} \\
& \leq\left\{[f(a)]^{r}[g(a)]^{r}\left(\int_{0}^{1}(1-t)^{\frac{2}{r}} \mathrm{~d} t\right)^{r}\right. \\
& +\left([f(a)]^{r}[g(a)+\eta(g(b), g(a))]^{r}\right. \\
& \left.+[g(a)]^{r}[f(a)+\eta(f(b), f(a))]^{r}\right)\left(\int_{0}^{1}[t(1-t)]^{\frac{1}{r}} \mathrm{~d} t\right)^{r} \\
& +\left([f(a)+\eta(f(b), f(a))]^{r}[g(a)+\eta(g(b), g(a))]^{r}\right)\left(\int_{0}^{1} t^{\frac{2}{r}} \mathrm{~d} t\right)^{r} \\
& =\left([f(a)]^{r}[g(a)]^{r}+[f(a)+\eta(f(b), f(a))]^{r}[g(a)+\eta(g(b), g(a))]^{r}\right)\left(\int_{0}^{1} t^{\frac{2}{r}} \mathrm{~d} t\right)^{r} \\
& +\left([f(a)]^{r}[g(a)+\eta(g(b), g(a))]^{r}\right. \\
& \left.+[g(a)]^{r}[f(a)+\eta(f(b), f(a))]^{r}\right)\left(\int_{0}^{1}[t(1-t)]^{\frac{1}{r}} \mathrm{~d} t\right)^{r} \\
& =\left\{M(a, b)\left(\frac{r}{r+2}\right)^{r}+N(a, b)\left(\beta\left(\frac{1}{r}+1, \frac{1}{r}+1\right)\right)^{r}\right\}
\end{aligned}
$$


which is the required result.

\section{ACKNOWLEDGEMENTS}

The authors would like to thank the Rector, COMSATS University Islamabad, Pakistan, for providing excellent research and academic environments.

\section{REFERENCES}

[1] G. D. Anderson, M. K. Vamanamurthy and M. Vuorinen, Generalized convexity and inequalities, J. Math. Anal. Appl, 335(2007), 1294-1308.

[2] M. Alomari, M. Darus and S. S. Dragomir, New inequalities of Simpson's type for s-convex functions with applications, RGMIA Res. Rep. Coll, 12 (4)(2009).

[3] C. Baiochi and A. Capelo, Variational and Quas-Variational Inequalities, Wiley, New York, (1984).

[4] J. Crank, Free and Moving Boundary Problems, Clarendon Press, Osford, UK, (1984).

[5] G. Cristescu, L. Lupsa, Non-connected Convexities and Applications, Kluwer Academic Publishers, Dordrechet, Holland,(2002).

[6] M. R. Delavar and S. S. Dragomir, On $\eta$-convexity, Math. Inequal. Appl, 20(1)(2017), 203-216.

[7] S. S. Dragomir and C. E. M. Pearce, Selected topics on Hermite-Hadamard inequalities and applications, Victoria University, Australia, (2000).

[8] R. Glowinski, J. L. Lions and R. Tremolieres, Numerical Analysis of Variational Inequalities, North-Holland, Amsterdam, (1981).

[9] M. E. Gordji, M. R. Delavar and M. D. Sen, On $\varphi$ convex functions, J. Math. Inequal, 10(1)(2016), 173-183.

[10] M. E. Gordji, M. R. Delavar and S. S. Dragomir, An inequality related to $\eta$-convex functions (II), Int. J. Nonlinear Anal. Appl, 6(2)(2015), 27-33.

[11] P. M. Gill, C. E. M. Pearce, J. Pecaric, Hadamards inequality for r-convex functions, J. Math. Anal. Appl, 215(1997), 461470.

[12] J. Hadamard, Etude sur les proprietes des fonctions entieres et en particulier dune fonction consideree par Riemann, J. Math. Pure. Appl, 58(1893), 171-215.

[13] C. Hermite, Sur deux limites d'une integrale definie, Mathesis, 3(1983), 82.

[14] D. H. Hyers and S. M. Ulam, Approximately convex functions, Proc. Amer. Math. Soc, 3(1952), 821-828.

[15] C. P. Niculescu and L. E. Persson, Convex Functions and Their Applications. Springer Verlag, New York, (2006).

[16] M. A. Noor, On Variational Inequalities, PhD Thesis, Brunel University, London, UK, (1975).

[17] M. A. Noor, General variational inequalities, Appl. Math. Letters, 1(1988), 119-121.

[18] M. A. Noor, New approximation schemes for general variational inequalities, J. Math. Anal. Appl, 251(2000), 217-230.

[19] M. A. Noor, Some developments in general variational inequalites, Appl. Math. Comput. 152(2004), 199-277.

[20] M. A. Noor and K. I. Noor, Harmonic variational inequalities, Appl. Math. Inform. Sci. 10(5)(2016), $1811-1814$.

[21] M. A. Noor, K. I. Noor and Th. M. Rassias, Some aspects of variational inequalities, J. Comput. Appl. Math. 47(1993), 285-312.

[22] M. A. Noor, K. I. Noor and M. U. Awan, Some new estimates of Hermite-Hadamard inequalities via harmonically convex functions, Le Mathematiche, LXXI(II)(2016), 117-127. 
[23] M. A. Noor, K. I. Noor, M. U. Awan and F. Safdar, On strongly generalized convex functions, Filomat, 31(18)(2017), 5783-5790.

[24] M. A. Noor, K. I. Noor and F. Safdar, Generalized geometrically convex functions and inequalities, J. Inequal. Appl, 2017(2017):22.

[25] M. A. Noor, K. I. Noor and F. Safdar, Integral inequaities via generalized convex functions, J. Math. Computer, Sci, $17(4)(2017), 465-476$.

[26] M. A. Noor, K. I. Noor, S. Iftikhar, F. Safdar, Integral inequaities for relative harmonic $(s, \eta)$-convex functions, Appl. Math. Comp. Sci, 1(1)(2015), 27-34.

[27] M. A. Noor, K. I. Noor, S. Iftikhar and S. Safdar, Generalized $(h, r)$-harmonic convex functionsand inequalities, Inter. J. Math. Anal. 16(4)(2018),542-555.

[28] M. A. Noor, K. I. Noor and F. Safdar, Integral inequaities via generalized $(\alpha, m)$-convex functions, J. Nonlinear. Func. Anal, 2017, (2017), Article ID: 32.

[29] M. A. Noor, K. I. Noor, S. Iftikhar, Inequaities via $(p, r)$-convex functions, RAD, (2018).

[30] M. A. Noor, K. I. Noor and F. Safdar, New inequalities for generalized log $h$-convex function, J. Appl. Math. Inform, 36(3-4)(2018), 245-256.

[31] M. A. Noor, K. I. Noor, F. Safdar, M. U. Awan and S. Ullah, Inequaities via generalized $\log m$-convex functions, J. Nonlinear. Sci. Appl, 10(2017), 5789-5802.

[32] M. A. Noor, K. I. Noor and F. Safdar, Generalized r-convex functions and integral inequalities. Int. J. Anal. Appl, 16(2018).

[33] C. P. Niculescu, Convexity according to the geometric mean, Math. Inequal. Appl, 3(2)(2000), 155-167.

[34] N. P. N. Ngoc, N.V. Vinh, P. T. T. Hien, Integral inequalities of Hadamard type for $r$-Convex functions, Int. Math. Forum, 4 (35)(2009), 1723-1728.

[35] G. Stampacchia, Formes bilineaires coercivities sur les ensembles convexes, C. R. Acad. Sci. Paris, 258(1964), $4413-4416$. 